\title{
Redes asociativas, inmigración y participación. Una aproximación empírica a los vínculos entre capital social y participación asociativa.
}

\author{
Mireia Bolíbar Planas
}

\begin{abstract}
Centre d'Estudis Sociològics sobre la Vida Quotidiana i el Treball (QUIT) -
Institut d'Estudis del Treball (IET) - Universitat Autònoma de Barcelona ${ }^{1}$
\end{abstract}

\begin{abstract}
Resumen
El artículo expone los resultados de una investigación empírica con el objetivo de conocer la interrelación entre el capital social que poseen las asociaciones de inmigrantes y sus estrategias participativas. Se explora así el papel de las redes inter-organizativas de las asociaciones de inmigrantes en la configuración de los discursos de sus miembros dirigentes sobre la capacidad y legitimidad de las asociaciones, y del trabajo inter-asociativo, para facilitar la acción colectiva e incidir en el espacio público. Con una estrategia de investigación mixta (combinando la Teoría y Análisis de Redes Sociales con el Análisis del Discurso) se ha realizado un estudio de casos comparativo de cinco asociaciones con representatividad tipológica. Mediante cuestionarios egocéntricos y entrevistas semiestructuradas, se ha obtenido la representación cognitiva de los dirigentes asociativos sobre su red de relaciones con otras asociaciones. El análisis de los resultados muestra una clara correspondencia entre la orientación de los discursos y las formas de capital social reticular bonding y bridging (de cohesión e integración) de las asociaciones, aunque la relación e importancia de la Administración pública en las redes aparece como un factor aún más relevante en la configuración de estrategias con voluntad de incidencia y participación política de las asociaciones.
\end{abstract}

Palabras clave: Redes asociativas - Estrategias de participación - Capital social Asociaciones de inmigrantes.

\begin{abstract}
The paper presents the results of an empirical dissertation done in order to know the connections between the social capital owned by immigrant organizations and its participatory strategies. It explores the role of its organizational networks in civil society on the configuration of the discourse of its leading members about migrant participation. The investigation has been carried out through the case study approach by comparing five typological representative organizations in the field of immigration. The object of study has been considered under the conceptual and operative frame of the Theory and Analysis of Social Networks combined with the Content Analysis. Through egocentered network questionnaire and semi-structured interviews it was possible to obtain the ego's cognitive representation of the relationships that shape the associations' network. Results show a correspondence between the orientation of the discourses and the organizations' type of social
\end{abstract}

\footnotetext{
${ }^{1}$ Beneficiaria del programa FPU del Ministerio de Educación.

Enviar correspondencia a Mireia.Bolibar@uab.cat
} 
capital (cohesive, bonding or integrating, bridging). Nevertheless, the relationship and importance of the Public Administration on the relational network appears as an even more important factor in the determination of the organizations' will to influence and participate on the political matters of the local community.

Key words: Organizational networks - Participatory strategies - Social capital Migrant associations.

La participación social y política de los inmigrantes, desde una noción amplia de los derechos políticos, de participación o de intervención en la vida pública, incluye todas aquellas manifestaciones de la llamada libertad positiva o democrática (De Lucas, 2008); tanto aquellos comprendidos en una visión más restrictiva de la participación política, es decir, el derecho al sufragio activo y pasivo y el acceso a cargos públicos, como aquellos de naturaleza participativa ${ }^{2}$. Es por este motivo que el asociacionismo inmigrante, aun no siendo un fenómeno nuevo está adquiriendo una relevancia emergente, tanto por su creciente importancia en número o volumen en la sociedad catalana como por su significación democratizadora en el campo de la participación cívica y política; pues en un contexto de exclusión de sus participantes del estatus de ciudadanía impera la búsqueda de nuevas formas de participación en la esfera pública ${ }^{3}$.

Aún así, la participación asociativa de la población inmigrante ha generado en los últimos años un debate en torno a sus funciones y potencialidades como herramienta de inclusión e integración. Por un lado, se argumenta que esta forma de asociacionismo puede favorecer la visibilización de este colectivo y sus necesidades, incrementar el contacto de sus miembros con el tejido asociativo en su conjunto y aumentar la participación de sus miembros en las políticas públicas y los asuntos comunitarios, propiciando que estos se conviertan en sujetos, y no sólo objetos, de la intervención y transformación social.

\footnotetext{
2 Partimos de una concepción amplia del concepto de participación, que no se restringe a las instituciones políticas formales. Se entiende que la participación es "la expresión pública del carácter cívico, económico, social y cultural inherentes a la propia condición de ciudadano dentro del sistema democrático" (Martín Pérez, 2004), así como el "conjunto de derechos, más allá del sufragio, cuyo reconocimiento y ejercicio posibilita a las comunidades (de inmigrantes) la intervención en la vida pública y la expresión de sus intereses, demandas y reivindicaciones" (De Lucas, 2008: 17).

3 Según ATIME (Asociación de Trabajadores Inmigrantes Marroquíes en España) (2008), "la importancia de la significación de las asociaciones de inmigrantes, formadas y gestionadas mayoritariamente por población inmigrante, recae directamente en el derecho y la necesidad de autorepresentación de los colectivos implicados como fórmula para completar un mapa asociativo y de participación ciudadana lo más representativo y variado posible".
} 
Sin embargo, por otro lado, las asociaciones de inmigrantes no siempre satisfacen estas características. Estas también pueden crear estructuras paralelas a las asociaciones existentes, propias y exclusivas para la población inmigrada, derivando en una "guetización asociativa de la minoría" (Morell, 2005), que bloquee la normalización de la participación de sus miembros en cualquier otro ámbito de participación y surque una fractura (cleavage) entre la población autóctona e inmigrante en el campo del asociacionismo ${ }^{4}$.

El objetivo de este artículo es explorar el papel y la importancia de las redes interasociativas de las asociaciones de inmigrantes (es decir, su capital social) en la configuración de sus estrategias participativas y la forma en que orientan su actividad. Se parte de la hipótesis de que las redes asociativas son un elemento esencial en la definición del tipo de impacto que estas puedan tener en la comunidad.

Para tal efecto, en primer lugar, en el artículo se caracterizan y exponen la amplia diversidad de funciones que realizan estas organizaciones; se debaten los elementos que dan importancia a las redes que estos actores crean en el medio asociativo e institucional; y se expone una conceptualización del capital social que nos permite aproximarnos de forma empírica a estas redes de colaboración interasociativa entendidas desde el punto de vista del recurso que suponen para quienes lo detentan. En segundo lugar, se plantea la investigación empírica, que trata vincular las redes inter-asociativas con las estrategias de participación de estas asociaciones $^{5}$. Para acabar, se presenta una reflexión sobre los mecanismos explicativos de esta relación así como algunas implicaciones de los resultados obtenidos.

\section{El asociacionismo inmigrante, y el papel en él de las redes asociativas y el capital social}

\section{El asociacionismo y la participación de la población inmigrante}

Las organizaciones del ámbito asociativo y concretamente también las asociaciones de inmigrantes son una realidad plural y heterogénea, en la que se realizan un

\footnotetext{
${ }^{4}$ En este artículo se hace uso de este término sin la connotación de exclusión, pobreza o marginación, sino para remitir a la idea de la concentración y 'separación'.

5 Entendiendo asociación como "un grupo formalmente organizado y con un nombre, en el que la mayoría de sus miembros-ya sean personas $u$ organizaciones-no se ven recompensados económicamente por su participación" (Knoke, 1986:2, en Montero, Font y Torcal (eds), 2006).
} 
amplio abanico de actividades con diferentes funciones. Warren (2001), distingue tres grandes tipos de consecuencias de las asociaciones: los efectos en sus propios miembros ("developmental effects"), su contribución a crear espacio público y lo que él Ilama "efectos institucionales", es decir, aquellos efectos provocados por las asociaciones en las instituciones de gobierno. En base a esta distinción, y a la vez acercándonos a las diferentes funciones ('guetizador/a' o integrador/a) atribuidas a las asociaciones, en base a sus destinatarios, y por tanto, sus efectos, se pueden clasificar los objetivos de las asociaciones de inmigrantes en dos tipos: por un lado, aquellos orientados a los miembros de la asociación y ceñidos a la consecución de beneficios para estos (es decir, el intragrupo), entre los que se encuentran los "developmental effects"; y por otro lado, los orientados al exogrupo, es decir, abiertos a la comunidad en general y no sólo a los miembros de la asociación, entre los cuales hay los "efectos institucionales" y la creación de espacio público.

Así, por un lado las asociaciones de inmigrantes responden a la necesidad humana de reunirse, dialogar, intercambiar afectos, inquietudes y experiencias, formar parte y participar en un grupo, etc. En el marco del proceso migratorio, el asociacionismo es una forma de superar el aislamiento social que en muchos casos provoca la migración en un contexto desconocido y un entorno diferente, fruto de la ruptura de vínculos debido a la migración. Facilita la acogida psicológica, fomenta la sociabilidad, el intercambio de experiencias, y disponer de referentes de integración (Morell, 2005). Molina et.al. (2008) exponen que el espacio de interacción que las asociaciones conforman tiene importancia en la conformación de los patrones de cambio en las redes personales de los inmigrantes, y por lo tanto, ejerciendo una influencia sobre el proceso de aculturación - concretamente, los autores apuntan al efecto "involutivo" de las asociaciones de compatriotas sobre este proceso -. También es destacable el papel que pueden tener en la creación y potenciación de una red de relaciones entre los residentes que actúe como protección ante situaciones de necesidad (Garreta, 1998). Asimismo, las asociaciones de inmigrantes a menudo también prestan servicios como el de la enseñanza del idioma del país receptor, asesoramiento y orientación sobre los servicios del país de acogida, y el mantenimiento de la cultura de origen y la transmisión de ésta a la segunda generación.

Por otro lado, las asociaciones de inmigrantes pueden ejercer funciones orientadas a la comunidad, como por ejemplo la vertebración de la vida social y deportiva de 
la comunidad, la sensibilización sobre el reconocimiento y visibilización de la diversidad (Casey, 1995), y la información y difusión de la propia cultura y tradiciones para darla a conocer como forma de lucha contra el racismo y la discriminación por motivos culturales (Garreta, 1998). Además, según Rex (1994), las organizaciones étnicas son un recurso para la lucha y la negociación de su participación social e integración afectiva en igualdad de oportunidades en la sociedad de acogida. Estas asociaciones, entendidas como una forma de vertebrar la minoría y de defender sus intereses como base para la acción colectiva, pueden actuar como catalizadoras del proceso de integración social y de su negociación, así como de su participación política (Garreta, 1998). Como ya afirmó Tocqueville (en Garreta, 1998), la tendencia al asociacionismo político, social, religioso y económico es una forma de representación de los intereses de los diferentes grupos existentes en una sociedad, y también un mecanismo de información y de participación en la vida política. En definitiva, el papel de las asociaciones es político al constituirse en fuentes de poder e influencia pública, de manera que pueden convertirse en generadoras activas, junto con otros actores, de esfera pública (Habermas, 1989), y modificar la cultura política del dominio público (Rex, 1994).

\section{Las redes inter-asociativas}

Siguiendo a De Lucas (2008), la plena inclusión de los extranjeros en los diferentes circuitos de la política y el ejercicio de la praxis ciudadana encuentra algunos límites, no sólo derivados tanto de la legitimación coyuntural del estatus quo de exclusión política parcial o total de los extranjeros, sino también del ejercicio de los derechos de participación. Se trata de obstáculos imputables a las condiciones que los países de acogida ofrecen a las comunidades de inmigrantes para el desarrollo de las actividades políticas o asociativas, así como a las dinámicas de la acción política y asociativa de los colectivos de extranjeros y a la relación de las organizaciones de inmigrantes con las estructuras de las instituciones, con las organizaciones de interés local y con las otras organizaciones de inmigrantes. En esta coyuntura las redes asociativas se vislumbran como un elemento clave en la definición de las funciones e implicaciones del asociacionismo inmigrante.

Las organizaciones, al igual que los individuos, pueden encontrarse en una situación de vulnerabilidad relacional sea por una situación generada por la ausencia o debilidad de los vínculos de inserción comunitaria, caracterizada por el aislamiento o la debilidad de la red social de apoyo, o bien por la integración en una subred marginalizada (Bonet, 2006). Contrariamente, sin embargo, las organizaciones 
también pueden establecer contactos, generar capital social activamente y elaborar estrategias de fortalecimiento de sus vínculos. Este networking y la creación de coaliciones es un elemento clave (aunque no condición suficiente) de los procesos de potenciación comunitaria (empowerment), es decir, "procesos dinámicos de adquisición de recursos, poder o influencia" (Maya, 2004: 11).

Las condiciones bajo las cuales emergen las redes de participación y colaboración asociativas, según Charbonneau y Simmard (2005) son la existencia de problemas que superan las capacidades de acción de cada asociación individualmente; las temáticas intrínsecamente transversales que superan los objetivos de las asociaciones particulares, la necesidad de anticiparse a situaciones que puedan afectar a la comunidad, o bien la necesidad de promoción de políticas o programas de acción conjuntos. Las redes inter-asociativas muestran un estadio intermedio de la articulación social, con un elemento identitario pero fluido y compatible con la existencia de varias comunidades que pueden compartir objetivos en un momento determinado y ofrecen la posibilidad de vincular movimientos, ciudadanía, asociaciones y grupos diversos en procesos de solidaridad y reciprocidad focalizados en objetivos concretos (Riera, C. en Martí, Pascual y Rebollo, 2005).

En algunas experiencias que relatan Blanco y Gomà, (2002) se constata que la capacidad de incidencia de las redes inter-asociativas en la política es bastante significativa en la dimensión simbólica, en la capacidad de generar discurso, establecer debate e introducir conceptos en la arena política, movilizando los medios de comunicación para generar opinión pública.

En la vertiente más instrumental, lo que constatan González y Morales (2006) en un estudio de las asociaciones de inmigrantes de Madrid, es que el hecho de pertenecer a una plataforma o red de organizaciones es un factor relacionado con un aumento significativo de la participación efectiva en el proceso político, lo que hace pensar en la posibilidad de que los contactos más o menos estables con otras asociaciones del entorno son un buen canal de comunicación que facilita la visibilidad pública de las asociaciones de inmigrantes. Martín Pérez (2004) destaca también la potencialidad de las asociaciones de inmigrantes y especialmente de la relación entre organizaciones para funcionar como una red de intereses que se vuelcan en el campo de las reivindicaciones ante el Estado y en el desarrollo de "nuevas formas" de participación política. 
El contexto institucional en el que se produce la participación asociativa tiene como referente la gobernanza, la cual, como tipo ideal de modelo de gobierno, comprende las lógicas y prácticas de interdependencia que se establecen entre los actores dentro y entre los diferentes niveles de gobierno (gobierno multinivel). Los gobiernos, desde esta concepción ideal de gobernanza, promueven, aceptan y llevan a cabo formas de organización de gobierno basadas en la aceptación de varios niveles de interacción; establecen "complicidades" que permiten sumar recursos y estrategias de actuación, incorporando la sociedad civil en las tareas de gobierno; y propiciando escenarios donde los actores locales se relacionen, se conozcan y colaboren en la persecución del bien común (Subirats, 2002).

Sin embargo, críticas realizadas al modelo de la gobernanza, además de debatir sobre la asimetría entre las organizaciones de la sociedad civil y la administración pública, o la falta de transparencia y representatividad democrática de los actores que participan en la gestión pública, señalan el hecho de que ni todos los agentes colectivos o grupos sociales son iguales en capacidad de intervención, ni intervienen en todas las materias. "En sociedades donde impera la desigualdad entre los actores colectivos no es posible disimular la situación desequilibrada en la que se encuentran: ni todos cuentan con los mismos recursos ni con las mismas oportunidades" (Vallès, 2000). La capacidad de generar una red de contactos en el nivel organizativo y comunitario es uno de estos recursos desigualmente distribuidos. Como afirma Stoker (2002), el capital social es un recurso cuya presencia es fundamental para facilitar la acción individual y colectiva. Según el autor, generalmente las redes de información, confianza y normas (que son un soporte esencial para la cooperación y la acción colectiva) están desigualmente distribuidas, de manera que los pobres en redes de trabajo juegan con una gran desventaja a la hora de participar en el nuevo ámbito del gobierno local. Aquellos que carecen del acceso al Capital Social se pueden encontrar al margen de la nueva política y de las formas de producción social. En definitiva, las diferencias en capital social generan desigualdades sustanciales en la acción social y política.

\section{El capital social en el nivel meso-social}

El capital social es un concepto complejo sobre el que se ha escrito ampliamente. Sin entrar a describir el debate generado en su conceptualización (véase, por ejemplo, Portes, 1998 o Lozares, 2011), cabe apuntar que en esta investigación nos hemos alejado de concepciones sobre el capital social de tipo "atributivo"entre las que podemos encontrar las obras de Putnam $(1993,2000)$ - es decir, que 
conceptualizan el Capital Social como un contenido cualitativo atributivo que, siendo producto de las relaciones sociales, es un recurso que constituye y desarrolla la comunidad (Martí y Lozares, 2008), especialmente a partir de la implicación en asociaciones voluntarias y organizaciones cívicas. En esta investigación, adoptamos el enfoque reticular del capital social ${ }^{6}$, entendiendo el Capital Social como un recurso centrado y distribuido en la estructura de las relaciones o redes sociales (Lozares, 2003). De este modo, se han estudiado las redes asociativas fruto de las prácticas de interacción, colaboración y participación conjunta entre las organizaciones como una manifestación o indicador del capital social de las asociaciones estudiadas, en su componente relacional. El capital social tiene su expresión en diferentes niveles de la realidad social; en la investigación que se presenta se ha focalizado el análisis sobre el entramado de relaciones entre organizaciones locales, centrando la atención en el capital social que desarrollan las asociaciones de inmigrantes en el nivel meso social (en el plano organizacional, en las estructuras intermedias entre los individuos y las grandes instituciones).

En línea con conceptualizaciones como la de Putnam (2000) o la de Woolcok y Narayan (2000), en base a la forma de distribución de las relaciones se distinguen en esta investigación dos tipos de capital social que se produce en este entramado de relaciones: el capital social de tipo bonding y el de tipo bridging. El primero, "de unión", de naturaleza cohesiva y endogámica, vincula horizontalmente nodos homogéneos, (pertenecientes a un mismo grupo social o "hacia dentro"). Los contenidos de las relaciones de tipo bonding, aunque se suelen asociar más a la confianza, pueden ser diversos. Se puede hablar de relaciones bonding de carácter económico, informativo, de apoyo mutuo material o instrumental, etc.; pueden contener un carácter más objetivado y material, o cognitivo como son las relaciones de confianza, vínculos identitarios o de valores (Lozares et al, 2011). En el ámbito de la participación, el capital social bonding facilita la cohesión, la identificación con un colectivo referente y la creación y mantenimiento de apoyos y ayudas directas.

En cambio, el capital social bridging, los lazos "que tienden puentes", de tipo más expansivo y exogámico, vincula nodos heterogéneos (pertenecientes a grupos sociales diferentes, es decir, relaciones "inter"-grupos). También sus contenidos pueden ser diversos, pero en general facilita una mayor integración relacional con el conjunto de grupos sociales (en este caso, el conjunto de la red comunitaria de

\footnotetext{
${ }^{6}$ Aproximación propia en Burt (1997), Lin (1999), Granovetter (1973) y más lateralmente a Bourdieu (1986) y Coleman (1998).
} 
asociaciones del municipio o territorio que se considera), así como la consecución de recursos diversos.

\section{La investigación empírica: El objeto de estudio y el modelo de análisis}

El objetivo de la investigación empírica ha sido analizar la interrelación que se produce entre, por un lado, las redes inter-asociativas de las asociaciones de inmigrantes (es decir, su capital social) y, por el otro, las prácticas y las percepciones de los dirigentes asociativos sobre la acción colectiva. Dejamos así a un lado indagar sobre el impacto de la participación asociativa en el capital social de los individuos que participan en ellas (cuestión que ha sido tratada en otras investigaciones, como Glanville, 2004; Lauer y Yan, 2010; o Bolíbar, 2011).

Se parte de la hipótesis de que las redes inter-asociativas son un elemento esencial en la definición de las percepciones de las personas inmigradas sobre las funciones del movimiento asociativo en la participación e intervención comunitaria, siendo estas percepciones la base sobre la que configuran sus estrategias participativas. Es decir, partimos de la idea de que existe una relación bidireccional entre la configuración de las redes inter-asociativas y dichas percepciones.

Para responder a las preguntas de la investigación se ha planteado un trabajo de campo (localizado en una ciudad del área metropolitana de Barcelona) que lleva a cabo un estudio comparativo de cinco asociaciones ${ }^{7}$ de un mismo territorio, con representatividad tipológica, de perfiles y características diferentes (en cuanto a su constitución, el tipo de actividad realizada y su relación con la administración pública $)^{8}$. Concretamente, se ha analizado las características de la entidad, su red de relaciones asociativas (relaciones de cooperación con otras asociaciones -de cualquier ámbito de actuación-, federaciones o entidades de segundo grado e instituciones públicas ${ }^{9}$ ), y el discurso de sus dirigentes.

\footnotetext{
7 Incluyendo tanto las entidades de inmigrantes -formadas como mínimo por un 50\% de población inmigrante tanto en la junta directiva como en el total de sus miembros- como las que trabajan para inmigrantes.

8 Aunque sean organizaciones de un mismo territorio (decisión que se ha tomado para evitar la influencia de diferentes políticas municipales de asociacionismo o inmigración sobre los discursos), se trata de redes egocentradas que no permiten ver la posición de las diferentes organizaciones estudiadas en una única red del municipio.

9 La pregunta generadora ha sido la siguiente: Podría indicar si su asociación ha establecido alguna relación o bien ha colaborado con alguna otra entidad o institución, en los últimos 2 años? Esta relación puede ser de cualquier tipo, desde pasar información, firmar manifiestos o declaraciones conjuntas, 0
} 
Para ello, se ha planteado una aproximación al objeto de estudio mediante una estrategia de investigación multimétodo, combinando las técnicas de investigación de la Teoría y Análisis de Redes Sociales con el análisis categorial de contenido. Para tal efecto se han utilizado los programas Egonet, Ucinet y Atlas.ti. Los métodos de obtención de la información que se han utilizado para responder las preguntas de investigación y validar las hipótesis, han constado, en primer lugar, de la realización de un cuestionario egocéntrico de las asociaciones para caracterizar y analizar las redes asociativas egocentradas de éstas (concretamente, la representación cognitiva de ego sobre la red de relaciones entre los alteri que configuran su red). Se ha adoptado una visión de la red personal como el conjunto de las relaciones que mantiene un agente (en el caso de estudio, una entidad asociativa) con otros actores (asociaciones e instituciones) que conforman su comunidad o grupo. Se parte de la consideración que el estudio de caso de una red social egocéntrica es una herramienta para obtener información no sólo del ego en sí, sino sobre el conjunto de la sociedad que condiciona la configuración concreta de la red social (Molina et.al., 2008).

En segundo lugar, se ha realizado una entrevista semiestructurada a un dirigente asociativo de cada una de las entidades elegidas. En estas entrevistas se ha buscado obtener un discurso vinculado a la práctica en la propia asociación y la red asociativa (con la ayuda de la visualización de los resultados de la encuesta), al mismo tiempo que se han planteado situaciones ideales con las que obtener un discurso que trascendiera la propia experiencia. La finalidad de estas entrevistas ha sido, concretamente, el de observar las percepciones de estos individuos sobre la naturaleza del asociacionismo inmigrante; la capacidad y legitimidad de las asociaciones para convertirse en actores sociales y políticos relevantes y legítimos (es decir, como grupo de interés o de presión); y el potencial del trabajo interasociativo para facilitar la acción colectiva (en los objetivos propios y los compartidos) e incidir en las políticas públicas locales.

En lo que respecta al análisis de las redes inter-organizativas, con la finalidad de facilitar la comparativa del capital social de las entidades analizadas, en base al análisis de la composición y estructura de sus redes de relación con otras entidades, asociaciones e instituciones diversas, se identifican y operativizan tres

asistir o participar en algún proyecto, actividad o reunión, hasta organizar y preparar actos / fiestas / campañas conjuntas, analizar conjuntamente problemas (comunes o no), apoyo logístico o financiero. Puede incluir Federaciones o plataformas, así como departamentos, instituciones o servicios municipales. 
indicadores o ejes de análisis complementarios el valor de los cuales clasifican las redes en los modelos o tipos diferentes:

- El capital social "bonding", cohesivo. Las entidades con capital social bonding se caracterizan por disponer de:

- Importante presencia de las asociaciones-Alteri de composición y naturaleza similares a la asociación-Ego.

- En el caso de existir subgrupos o componentes, estos se caracterizan por disponer de una baja relación exogrupo y de una composición interna también endogámica (con poca diversidad de ámbitos de trabajo y de nacionalidades).

- El capital social "bridging", expansivo o integrador. Las entidades con un capital social bridging se caracterizan por disponer de:

- Mayor presencia de asociaciones-Alteri de composición y naturaleza diferentes entre sí y con la asociación-Ego.

- Mayor presencia de Alteri vinculados al territorio.

- La intensidad de capital social es el tercer elemento que define la topología de redes egocentradas de las entidades, y que complementa las otras dos ${ }^{10}$. Es una entidad con un capital social elevado aquella en la que se encuentre:

- Elevada dimensión de la red, es decir, del nombre de nodos que la configuran.

- Elevada densidad, con nodos muy comunicados y relacionados entre sí.

- Baja jerarquía y alta igualdad en la posición de los nodos en la estructura de la red.

\footnotetext{
10 Es decir, la redes pueden disponer de mayor o menor capital social, el cual puede ser de tipo más o menos bonding, y más o menos bridging. La mayor presencia de capital social favorece la cohesión y la integración del capital bonding y bridging, respectivamente.
} 
- Estructura de la red cohesiva, con un elevado índice de cohesión interna, y baja distancia entre los Alteri. También elevada proximidad global.

- Baja centralidad (de grado) e intermediación (tango global como de los nodos más centrales).

- Densidad y cohesión de las cliques.

- Poca presencia y centralidad de la administración pública en la red.

\section{Los datos obtenidos}

Los resultados del análisis de los datos obtenidos mediante el trabajo de campo muestran la existencia de redes de colaboración, que a pesar de ser de diferentes tipos y niveles, nos llevan a afirmar la existencia de ciertas sinergias de colaboración interasociativa en la comunidad estudiada, y que, en algunos casos, vinculan grupos sociales heterogéneos.

Estos resultados, además, validan la existencia de una cierta correspondencia entre el capital social de las entidades estudiadas y los discursos de sus dirigentes relativos a las funciones de su entidad y del tejido asociativo en su conjunto, las potencialidades de la colaboración inter-asociativa, y la estructura de oportunidades de participación en la política municipal.

Para facilitar el análisis comparativo se presentan aquí, junto con una pequeña caracterización de las asociaciones, las redes de colaboración asociativa de las cinco organizaciones estudiadas que muestran su entorno relacional ${ }^{11}$. Con ellas se presentan también los elementos observados en los discursos de sus dirigentes, agrupados en seis ítems genéricos: la orientación (hacia el propio grupo o hacia la comunidad en general) de las actividades de la entidad; los elementos sobre los cuales se elabora un discurso crítico; la naturaleza de su voluntad de incidencia política; la relación de la organización con la Administración pública local; su visión respecto la legitimidad política de las asociaciones de inmigrantes; y finalmente su percepción sobre los beneficios de la red asociativa y el trabajo colaborativo en red. 
Tabla 1. Caracterización de las asociaciones estudiadas.

\begin{tabular}{|c|c|c|c|c|c|}
\hline Asociación & $\begin{array}{c}\text { Ámbito } \\
\text { actuación }\end{array}$ & $\mathbf{n}$ - miembros & $\begin{array}{c}\text { \% miembros } \\
\text { nacidos } \\
\text { extranjero }\end{array}$ & $\begin{array}{c}\text { Volumen } \\
\text { financiación } \\
\text { anual }\end{array}$ & $\begin{array}{c}\text { Origen } \\
\text { mayoritario }\end{array}$ \\
\hline A & trabajo social & 250 & 14 & $800000 €$ & $\begin{array}{c}\text { Catalunya, } \\
\text { España. }\end{array}$ \\
\hline B & religión & 50 & 90 & $\begin{array}{l}0 € \\
+ \text { servicios }\end{array}$ & Marruecos \\
\hline C & $\begin{array}{c}\text { integración } \\
\text { inmigración }\end{array}$ & 120 & 80 & $\begin{array}{c}3000 € \\
+ \text { servicios }\end{array}$ & $\begin{array}{c}\text { Bolivia y otros } \\
\text { Suramérica }\end{array}$ \\
\hline D & $\begin{array}{c}\text { integración } \\
\text { inmigración }\end{array}$ & 70 & 100 & $\begin{array}{c}0 € \\
\text { servicios }\end{array}$ & $\begin{array}{c}\text { Ecuador y otros } \\
\text { Suramérica }\end{array}$ \\
\hline E & $\begin{array}{c}\text { integración } \\
\text { inmigración }\end{array}$ & 25 & 80 & $\begin{array}{c}\text { Perú y otros } \\
\text { servicios }\end{array}$ & Suramérica \\
\hline
\end{tabular}

${ }^{11}$ En estas visualizaciones se observa únicamente el entorno relacional de Ego; Ego es invisible. 
Tabla 2. Resumen de los discursos de los dirigentes asociativos y gráficos de las redes de las organizaciones.

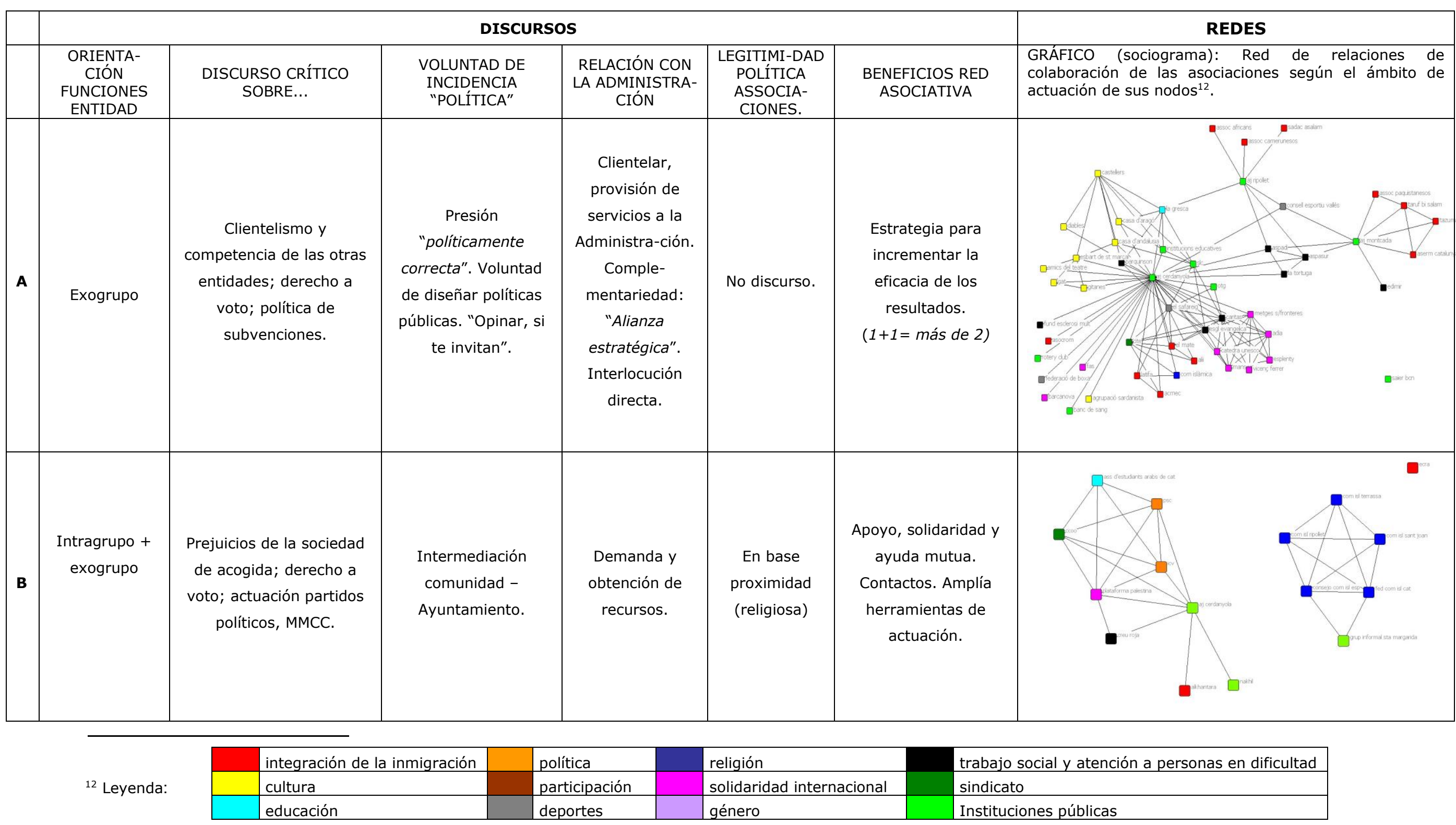




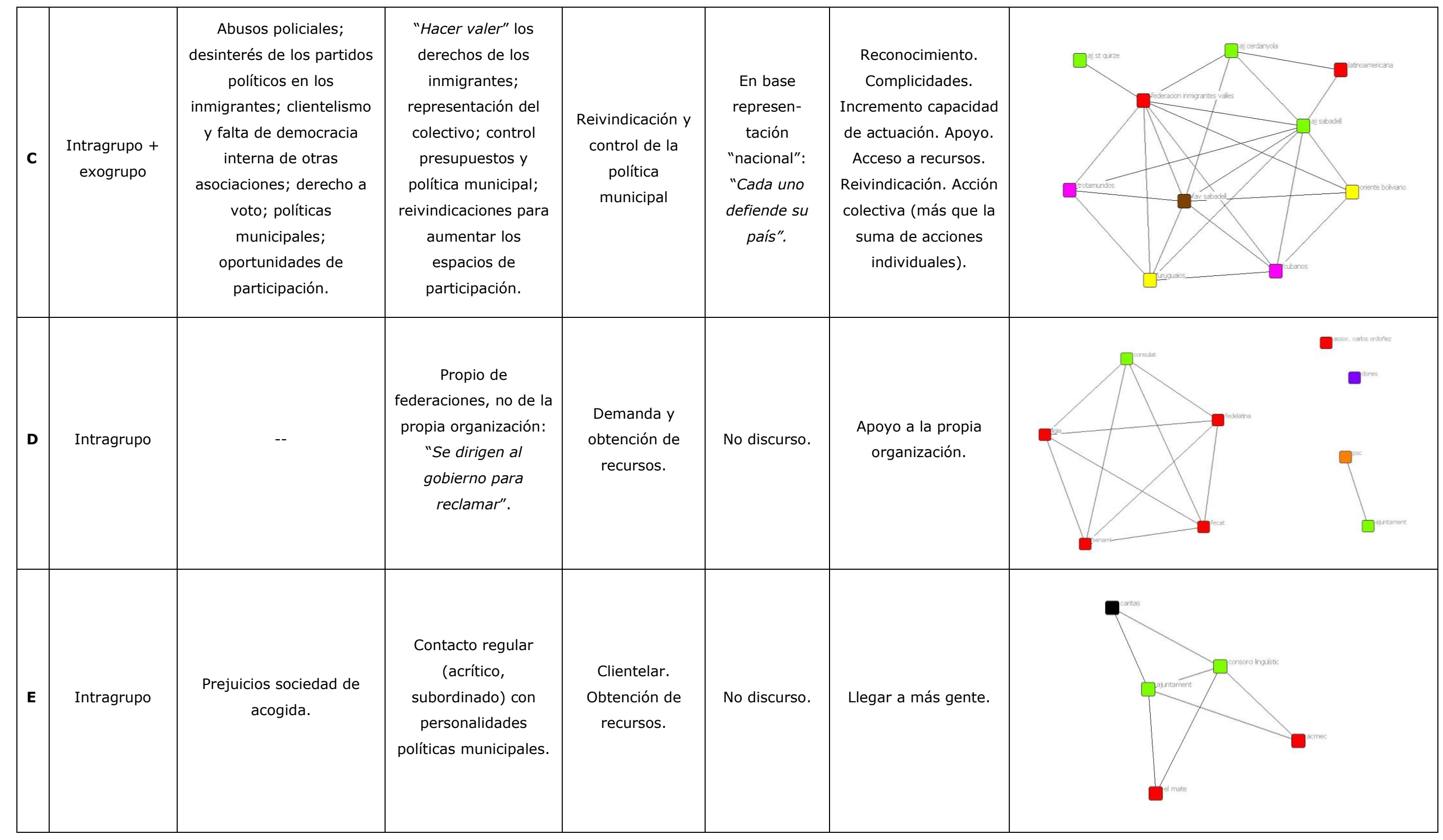


Finalmente, se presentan los indicadores de composición y estructura de las redes que, junto a la presentación gráfica de las mismas, permiten su caracterización (descripción del "perfil de la red"), y la clasificación de su Capital Social.

\begin{tabular}{|c|c|c|c|c|c|c|c|c|c|c|c|c|c|}
\hline \multirow{3}{*}{ 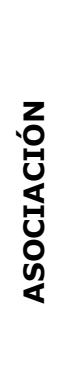 } & \multicolumn{10}{|c|}{ Indicadores de composición y estructura de las redes } & \multirow{2}{*}{\multicolumn{3}{|c|}{ Perfil de la Red }} \\
\hline & & \multicolumn{3}{|c|}{ Estructura } & \multicolumn{6}{|c|}{ Composición } & & & \\
\hline & $\begin{array}{l}\text { No de } \\
\text { nodos }\end{array}$ & $\begin{array}{l}\text { Cohesión } \\
\text { (basada en } \\
\text { la distancia } \\
\text { o cercanía) }\end{array}$ & $\begin{array}{l}\text { Centrali- } \\
\text { dad de } \\
\text { grado } \\
\text { (global) }\end{array}$ & $\begin{array}{l}\text { Interme- } \\
\text { diación } \\
\text { (global) }\end{array}$ & $\begin{array}{c}\% \text { Alteri } \\
\text { del mismo } \\
\text { ámbito que } \\
\text { ego }\end{array}$ & $\begin{array}{c}\mathrm{N}^{\circ} \text { de } \\
\text { ámbitos } \\
\text { presentes } \\
\text { en la red }\end{array}$ & $\begin{array}{c}\% \text { Alteri } \\
\text { extran- } \\
\text { jeros }\end{array}$ & $\begin{array}{l}\text { \% Alteri de la } \\
\text { misma } \\
\text { proceden-cia } \\
\text { que ego }\end{array}$ & $\begin{array}{c}\% \text { Alteri } \\
\text { del } \\
\text { municipio }\end{array}$ & $\begin{array}{l}\% \text { Alteri } \\
\text { del } \\
\text { municipio } \\
+ \text { alteri } \\
\text { comarca }\end{array}$ & $\begin{array}{l}\text { DIMEN- } \\
\text { SIÓN }\end{array}$ & $\begin{array}{l}\text { DIVERSI- } \\
\text { DAD } \\
\text { (en tipo de } \\
\text { asociacio- } \\
\text { nes) }\end{array}$ & $\begin{array}{l}\text { CAPITAL } \\
\text { SOCIAL }\end{array}$ \\
\hline \multirow[b]{2}{*}{ A } & \multirow[b]{2}{*}{52} & \multirow[b]{2}{*}{0.458} & \multirow[b]{2}{*}{$69.73 \%$} & \multirow[b]{2}{*}{$74.68 \%$} & \multirow[b]{2}{*}{15,4} & \multirow[b]{2}{*}{10} & \multirow[b]{2}{*}{$25,0 \%$} & \multirow[b]{2}{*}{$75,0 \%$} & \multirow[b]{2}{*}{$67,0 \%$} & \multirow[b]{2}{*}{$77,0 \%$} & \multirow[b]{2}{*}{ Grande } & \multirow[b]{2}{*}{ Muy elevada } & Alto \\
\hline & & & & & & & & & & & & & Bridging \\
\hline \multirow[b]{2}{*}{ B } & \multirow[b]{2}{*}{16} & \multirow[b]{2}{*}{0.335} & \multirow[b]{2}{*}{$24.76 \%$} & \multirow[b]{2}{*}{$13.27 \%$} & \multirow[b]{2}{*}{31,3} & \multirow[b]{2}{*}{8} & \multirow[b]{2}{*}{$62,5 \%$} & \multirow[b]{2}{*}{$62,5 \%$} & \multirow[b]{2}{*}{$50,0 \%$} & \multirow[b]{2}{*}{$50,0 \%$} & \multirow[b]{2}{*}{ Mediana } & \multirow[b]{2}{*}{ Elevada } & Mediano \\
\hline & & & & & & & & & & & & & $\begin{array}{r}\text { Bonding+ } \\
\text { bridging }\end{array}$ \\
\hline \multirow[b]{2}{*}{$\mathrm{C}$} & \multirow[b]{2}{*}{10} & \multirow[b]{2}{*}{0.757} & \multirow[b]{2}{*}{$44.44 \%$} & \multirow[b]{2}{*}{$24.59 \%$} & & & & & & & Mediana- & & Mediano \\
\hline & & & & & 20 & 5 & $50,0 \%$ & $10,0 \%$ & $20,0 \%$ & $100,0 \%$ & pequeña & Mediana & Bridging \\
\hline D & 9 & 0.347 & $25 \%$ & $0 \%$ & 55,6 & 4 & $66,7 \%$ & $55,6 \%$ & $33,3 \%$ & $33,3 \%$ & Pequeña & Mediana-baja & $\begin{array}{c}\text { Mediano- } \\
\text { bajo }\end{array}$ \\
\hline & & & & & & & & & & & & & Bonding \\
\hline & & & & & & & & & & & & & Bajo \\
\hline $\mathrm{E}$ & 5 & 0.850 & $50.00 \%$ & $18.75 \%$ & 40 & 3 & $40,0 \%$ & $0,0 \%$ & $100,0 \%$ & $100,0 \%$ & Pequeña & Baja & Bonding \\
\hline
\end{tabular}

Tabla 3: Resumen y comparativa de los perfiles de las redes estudiadas. Indicadores sobre la composición y estructura de las redes. 
Llevando a cabo una comparativa entre los casos estudiados, se destaca lo siguiente: En primer lugar, se observa que las entidades que disponen de menor capital social, y con un capital social de tipo bonding, orientan las funciones de la propia asociación hacia el intragrupo (ayuda mútua, sociabilidad, capacitación y formación, reproducción de la cultura del país de origen, etc.), mientras que aquellas entidades que poseen capital social de tipo bridging, también tienen en consideración el exogrupo en sus funciones y objetivos (con programas dirigidos a la comunidad en general como por ejemplo visibilizar y dignificar el colectivo, romper prejuicios, denunciar los abusos policiales, etc.). Aun así, se observa en las organizaciones estudiadas que el capital social bridging está asociado con una apertura hacia el exogrupo sin que eso "difumine los límites" del intragrupo. Se sigue considerando el tejido asociativo ( $y$ especialmente la pertenencia de los miembros a las organizaciones que lo forman) segregado según el origen étnico, donde cada organización tiene sentido porque defiende a su grupo (bien sean sus compatriotas o su comunidad religiosa).

En segundo lugar, se observa como la existencia de capital social bonding se relaciona con un uso de la red de contactos interasociativos exclusivamente para el apoyo mutuo (ayuda económica, intercambio de información, etc.), mientras que la existencia de capital social bridging se entrevé como un elemento facilitador del uso de esta red para la constitución de grupos de presión e incidencia, en vistas a la participación en ámbitos externos a los de la propia entidad (como por ejemplo reivindicar derechos, controlar los presupuestos municipales, apoyar campañas de otras organizaciones o reclamar mayores espacios de participación).

Sin embargo, se observa que, a pesar del capital social del que dispongan, las entidades con mayor capacidad de interlocución con la administración tienen menos necesidad de "hacer uso" de la red, y establecen estrategias de participación más individuales. Por otro lado, las asociaciones con muy poco capital social se encuentran en situación de mayor vulnerabilidad relacional, en la que su dependencia de la administración pública es mayor (tiene un papel muy importante en su red de colaboraciones) y, por tanto, su discurso crítico y su voluntad de incidencia política es menor. Observamos pues, una clara relación entre la posición hacia los poderes públicos (ya sea por su capacidad de interlocución, o bien por la dependencia relacional) y la voluntad de incidencia y participación política. Estos resultados son congruentes con la realidad expuesta por Martín Pérez (2004): La posibilidad de las redes asociativas de colaboración con entidades de la misma naturaleza para constituirse como grupo de presión y de participación efectiva en el 
espacio público se ven dificultadas por la relación jerárquica y de dependencia de los poderes públicos que en muchos casos establecen las asociaciones con la administración pública, así como por la (más o menos explícita) competencia entre las asociaciones por el reconocimiento institucional.

Se desprende del análisis también la poca consideración, por parte de las organizaciones más endogámicas, de la territorialidad (la vinculación con las asociaciones e instituciones del territorio) como un elemento importante para el ejercicio de la participación (externa a la entidad). Se refuerza así la idea según la cual el capital social bridging tiende puentes y facilita la construcción de una realidad vinculada al mundo local.

Finalmente, en las asociaciones de inmigrantes, también se constata una posible relación entre la existencia de capital social bridging y la consideración de la legitimidad de la implicación política de las asociaciones.

Así, el análisis realizado valida la idoneidad de los tipos ideales de redes asociativas propuestos como referentes clasificatorios del panorama asociativo y participativo: El capital social bonding facilita la cohesión, la identificación con un colectivo referente y la creación y mantenimiento de apoyos y ayudas directas, mientras que el capital social bridging facilita la integración en el conjunto de asociaciones del municipio y en su idiosincrasia local, lo que facilita la participación de la asociación en ámbitos externos a los de la propia entidad, como son las cuestiones relacionadas con la participación política.

\section{Conclusiones}

El presente artículo constata la existencia de una relación bidireccional entre el capital social disponible y existente en una asociación en el ámbito meso social de relaciones de colaboración inter-asociativa y los discursos y estrategias de sus dirigentes hacia la participación social y política de las mismas. Se reafirma pues la idea de que el capital social de una asociación es una parte constitutiva importante de la misma, no un anexo complementario; "el capital social configura la identidad fundamental de todo sujeto social" (Lozares, 2003: 26).

Se planteaba al inicio de este trabajo si las asociaciones de inmigrantes producen un efecto integrador $o$ bien si crean estructuras paralelas que fomenten la "guetización asociativa" (Morell, 2005). A partir de este trabajo podemos reafirmar 
que existe una amplia diversidad de casos: Aquellas entidades que disponen de poco capital social y exclusivamente de capital social bonding se aproximan a una situación de guetización asociativa, es decir, el discurso que explica y justifica sus actividades y su razón de ser se focaliza únicamente en el 'intragrupo', sin buscar tener un impacto de mayor alcance en la comunidad (lo cual no significa que tenga un impacto negativo en ella), quedando así al margen de la "nueva política" y formas de producción social. Este fenómeno, sin embargo, no se produce en todos los casos; otras asociaciones también disponen de capital social bridging, el cual facilita su integración en el tejido asociativo del territorio y su participación efectiva en el espacio público. Así pues, frente a una visión unívoca tanto del asociacionismo inmigrante como de los efectos del capital social, hay que destacar la multiplicidad de formas y funciones que estos pueden adoptar.

Apunto la naturaleza dual del capital social distribuido y apropiado en la red, el cual, según Martí y Lozares (2008) tiene una vertiente fáctica (relaciones establecidas a partir de hechos manifiestos y externos), y otra socio-cognitiva (la distribución de representaciones, semánticas, discursos o espacios mentales compartidos que están en la base de las relaciones y la colaboración efectiva entre los agentes) como uno de los elementos que explican la interrelación entre las redes de colaboración inter-asociativa y los discursos de los dirigentes asociativos. Las representaciones cognitivas de la realidad social compartidas están en la base de las relaciones y la colaboración efectiva, a la vez que las identidades, normas y valores constituyen también el capital social generado en la interacción (Martí y Lozares 2008). El entramado de relaciones entre los individuos, asociaciones e instituciones puede favorecer o impedir el acceso a determinados recursos que influyen en sus actividades, comportamientos y cogniciones. Las redes de colaboración interasociativa facilitan así la transmisión y difusión de actitudes que favorecen diferentes tipos de participación.

Las redes de relaciones entre asociaciones adquieren relevancia por el hecho que permiten el mantenimiento de la propia identidad e independencia al mismo tiempo que incrementan las posibilidades de conseguir recursos informativos u otros, así como maximizan su capacidad de actuación. En el caso de las asociaciones de inmigrantes, que aun no siendo un fenómeno nuevo, recientemente ha experimentado un incremento considerable, las relaciones que estas establecen con su entorno denotan el proceso por el cual estas crean un espacio propio y "se hacen un lugar" en el espacio público. En este proceso inicial pueden aprovechar la colaboración inter-asociativa para el "aprendizaje" del savoir faire, el conocimiento 
de las instituciones y el juego institucional local; así como establecer alianzas y definir los límites del propio grupo.

Además, las estrategias de interacción que diseñan estos actores, bajo determinados intereses racionales y contextos condicionantes, muestran los procesos de integración o cohesión y clausura del colectivo de inmigrantes en la sociedad de acogida. Cabe destacar la importancia que se desprende también de este estudio del papel y funciones de la Administración Pública en el campo asociativo y en el contexto de las relaciones inter-asociativas; su actuación puede marcar las pautas de relación entre las asociaciones de un territorio. Así, estos procesos de integración y cohesión no pueden ser comprendidos sólo desde la óptica de los inmigrantes, sino que las interacciones entre asociaciones de autóctonos y de inmigrantes nos informan también sobre la sociedad de acogida, sus instituciones y dinámicas $y$, en definitiva, sus respuestas ante el fenómeno inmigratorio.

Finalmente, cabe destacar la pertenencia de la metodología utilizada, basada en el análisis de redes sociales, así como su complementación con los estudios del discurso. La utilización de métodos mixtos en esta investigación ha permitido el abordaje integral del objeto de estudio, posibilitando la contextualización de las estrategias participativas en su entorno relacional. Además, si bien el análisis de redes sociométricas para el estudio de las redes inter-organizativas en este caso habría permitido una visión más territorializada, conectando las realidades de cada asociación (que han sido estudiadas como independientes) para conocer las dinámicas compartidas que las vinculan, al mismo tiempo que habría proporcionado una información de la estructura de la red de mayor calidad (pues las representaciones cognitivas de los egos sobre la relación entre sus alteri a menudo está sesgada); el uso de redes egocentradas de estas organizaciones ha permitido profundizar en la realidad individual de cada asociación. Estas han permitido, por un lado, conocer las diferentes esferas que se cruzan en la colaboración interasociativa, permitiendo ver en conjunto la interdependencia y presencia de todos los territorios y realidades implicadas en la elaboración de estrategias participativas por parte de las asociaciones; y por el otro, hacer emerger redes "invisibles", ya que ciertas formas de capital social bonding, desde una aproximación exclusivamente sociométrica, hubiesen sido omitidas e interpretadas como signos de vulnerabilidad relacional. Sin embargo, la investigación aquí presentada también presenta algunas limitaciones, fruto de la extensión del trabajo de campo. Si bien futuras investigaciones en esta línea de trabajo pueden beneficiarse de esta 
exploración en el campo de estudio, un trabajo con mayor número de casos sería muy enriquecedor para ampliar la capacidad de comparativa entre tipo de asociaciones, colectivos y territorios (con diferentes estructuras de oportunidades políticas), con lo que afianzar las tendencias que aquí hemos apuntado.

\section{Referencias bibliográficas}

Blanco, I. y Gomà, R. (2002). Gobiernos locales y redes participativas. Barcelona: Ariel.

Bolíbar, M (2011). "Las asociaciones en las redes personales. ¿Mecanismo de integración de la población inmigrante?" Redes. Revista Hispana para el análisis de las redes sociales, no 20, (7) <http://revista-redes.rediris.es>.

Bonet, J. (2006). "La vulnerabilidad relacional: Análisis del fenómeno y pautas de intervención". Redes. Revista Hispana para el análisis de las redes sociales, no $11,(2)<$ http://revista-redes.rediris.es>.

Burt, R.S. (1997). "The contingent value of social capital", Administrative Science Quarterly, no 42, Pp. 339-365.

Casey, J. (1997). "Les associacions i la integració d'immigrants estrangers". Revista Catalana de Sociologia. No 6, Pp. 9-22.

Charbonneau y Simmard (2005) "Networks of community associations and collective social capital: a review of analyses and experiences". En AAVV (2005) Social Capital in Action. Thematic Policy Studies Report. Pri Project: Social capital as a public policy tool. Policy Research Initiative, Government of Canada.

Colectivo Política En Red. (2007). Repensar la política en la era de los movimientos y de las redes. Barcelona: Icària.

De Lucas, J (2007). "Integración política, participación y ciudadanía: un balance". Entelequia. Revista interdeisciplinar, no 4, Pp. 271-282. $<$ http://www.eumed.net/ entelequia>

De Lucas, J. (2008). Los derechos de participación como elemento de integración de los inmigrantes. Bilbao: Fundación BBVA. <http://fbbva.es/TLFU/dat/>

Garreta, J. (1998). "Minories ètniques, associacionisme i integració sociocultural". Papers. Revista de Sociologia, no 56. Pp 197-230.

Garreta, J. Et al. (2008). "Associacionisme i acomodació de les persones immigrades d'origen africà a Catalunya". Col-leció ciutadania i immigració, no 1 Pp: 103-118.

Glanville, Jennifer. (2004). "Voluntary Associations and Social Network Structure: Why organizacional location and type are important". Sociological Forum, no 19, N03.

González Ferrer, A. y Morales, L. (2006). "Las asociaciones de inmigrantes en Madrid. Una nota de investigación sobre su grado de integración política". Revista Española del tercer sector. Núm. 4. Pp 1-37. 
Granovetter, M. (1973). "The Strength of Weak Ties", American Journal of Sociology, 78 (6) Pp. 1360-1380.

Habermas, J. (1989), The Structural Transformation of the Public Sphere: An Inquiry into a Category of Bourgeois Society, Thomas Burger, Cambridge Massachusetts: The MIT Press.

Lauer, S.R., Yan, M.C. (2010). "Voluntary Association Involvement and Immigrant Network Diversity", International Migrations, En prensa.

Lin, N. (1999). "Building a network theory of Social Capital". Connections, no 22.

Lozares C. (2003). "Valores, campos y capitales sociales". Redes. Revista hispana para el análisis de redes sociales No 4. <www. redes-sociales.net>.

Lozares, C.; J. M. Verd, P. López-Roldán, J. Martí y J. L. Molina (2011). "Cohesión, Vinculación e Integración sociales como formas de Capital social", REDESRevista hispana para el análisis de redes sociales, No 20 (1) < http://revistaredes.rediris.es>.

Martí, J., Pascual, J. y Rebollo, O. (coords.). (2005). Participación y desarrollo comunitario en medio urbano. Experiencias y reflexiones. Madrid: IEPALA Editorial.

Martí, J., Lozares, C. (2008). "Redes organizativas locales y capital social: Enfoques complementarios desde el análisis de redes sociales". Portularia. Revista de Trabajo Social. Universidad de Huelva. , no 7, pp. 461-480.

Martín Pérez, A. (2004) "Las asociaciones de inmigrantes en el debate sobre las nuevas formas de participación política y de ciudadanía: reflexiones sobre algunas experiencias en España". Migraciones ,15: 113-143.

Maya Jariego, I. (2004). "Sentido de comunidad y potenciación comunitaria". Apuntes de Psicología, 22 (2), 187-211.

Molina, J.L., Lerner, J., Gómez Mestres, S. (2008). "Patrones de cambio de las redes personales de inmigrantes en Cataluña". Redes. Revista Hispana para el análisis de las redes sociales, n015, (4) <http://revista-redes.rediris.es>.

Montero J.R., Font, J., Torcal, M. (eds.). (2006). Ciudadanos, asociaciones y participación en España. Madrid: Centro de Investigaciones Sociológicas.

Morales, L., y Anduiza, E. (dir.) (2009). "Actituds, comportament polític i xarxes organitzatives dels immigrants a la ciutat de Barcelona" Jaume Bofill. Col-lecció Informes Breus, no 15.

Morell Blanch, A. (2005). "El papel de las asociaciones de inmigrantes en la sociedad de acogida: cuestiones teóricas y evidencia empírica". Migraciones, 17. Pp 111-142.

Portes, A. (1998). "Social capital: Its origins and applications in modern sociology." Annu. Rev. Sociol. 24: 1-24.

Putnam, R. (1993). Making Democracy Work:Civic Traditions in Modern Italy. Princeton: Princeton University Press. 
Putnam, R. (2000). Bowling Alone: The Collapse and Revival of American Community. New York: Simon and Schuster.

Rex, J, y Beatrice, D. (1994). Ethnic mobilisation in a multi-cultural Europe. Aldershot: Asgate Publishing Ltd.

Stoker, G. (2002): "El trabajo en red en el gobierno local: una meta deseable, pero... ¿es posible alcanzarla?", en Subirats, J. (coord.) Redes, Territorios y Gobierno. Nuevas respuestas locales a los retos de la globalización. Barcelona: Diputación de Barcelona. Xarxa de municipis. Colección Territorio y gobierno, no 1.

Subirats, J. (coord.) (2002). Redes, territorios y gobierno. Barcelona: Diputació de Barcelona.

Vallès, J.M. (2000). Ciencia Política. Una introducción. Barcelona: Ariel.

Veredas, S. (2003). "Las asociaciones de inmigrantes en España. Práctica clientelar y dependencia política". Revista Internacional de Sociología (RIS) n036: 207225.

Vertovec, S. (1999). "Minority Associations, networks and public policies: reassesting relationships", Journal of Ethnic and Migration Studies, 25(1), 21-42.

Warren, M. (2001). Democracy and Association. Princeton: Princeton University Press.

Woolcock y Narayan (2000). "Social Capital: Implications for Development Theory, Research, and Policy". World Bank Research Observer. No 15(2)

Zapata, R. (2000). "Inmigración e innovación política", Migraciones, n 8, pp. 7-58. 
